Hydraulic Engineering Repository

Ein Service der Bundesanstalt für Wasserbau

Wendt, Dietrich

Bewehrte Gründungspolster

Verfügbar unter / Available at:

https://hdl.handle.net/20.500.11970/106242

Vorgeschlagene Zitierweise / Suggested citation:

Wendt, Dietrich (1987): Bewehrte Gründungspolster. In: Mitteilungen der Forschungsanstalt für Schiffahrt, Wasser- und Grundbau; Schriftenreihe Wasser- und Grundbau 52. Berlin: Forschungsanstalt für Schiffahrt, Wasser- und Grundbau. S. 126-142. 


\section{Bewehrte Gründungspolster}

3.1. Kommentax zur Vorschrift der Staatlichen Bauaufsicht Nr. 120, Blatt 3 (Entwurf) "Bewehrte Erde - horizontal bewehrte Grundungspolster"

therblick

In der internationalen Literatur, z. B. $/ 14 /, / 15 /, / 16 /, / 17 /$, sind theoretische und experimentelle Ergebnisse zur Wirkungsweise und zum Verhalten von bewehrten Grindungspolstern veröffentlicht. Aufgrund der Veröffentlichungen kann erwartet werden, daß die tragfähigkeitserhöhende Wirkung von Grïndungspolstern in wenig tragfähigem Baugrund durch horizontale Bewehrungslagen aus zugfestem Material in erheblichem Maße verstärkt wird. Ähnlich wie bei Stüzwandkonstruktionen, die nach der Bauweise "Bewehrte Erde" errichtet wurden, beruht die Wirkung von bewehrten Grindungspolstern auf dern Zusammenwirken von Polstererdstoff ohne nennenswerte Zugfestigkeit aber mit Scherfestigkeit und Bewehrungselementen mit Zugfestigkeit und Grenzflächen-Reibungsfestigkeit gegenuber dem umgebenden Erdstoff. Die Nutzung der tragfähigkeitserhöhenden Wirkung bewehrter Grindungspolster setzt detalllierte Kenntnisse uber die Wirkungsmechanismen der einzelnen Konstruktionselemente voraus, um die Sicherheit aller Teile der Konstruktion nachweisen zu können. Deshalb wurden umfangreiche theoretische und experimentelle Untersuchungen mit bewehrten Grindungspolstern an der Wilhelm-Pleck-Universitat Rostock und von der Forschungsanstalt fur Schiffahrt, Wasser- und Grundbau Berlin durchgeführt. Die Modellfundamente, bis zur Größe von $75 \times 150 \mathrm{~cm}$, wurden Belastungen bis zu $1000 \mathrm{kN}$ ausgesetzt. Auf die Ergebnisse dieser Arbeiten soll hier jedoch nicht naher eingegangen werden. Sie sind in /29/, /30/ dargestellt.

Die gewonnenen Ergebnisse in Verbindung mit Angaben aus der internationalen Ilteratur sowie Erkenntnisse aus eigenen Forschungsarbeiten über Stützbauwerke nach der Bauweise "Bewehrte Erde" fanden thren Niederschlag in /31/. Diese Vorschrift erganzt zwei bereits verbindliche Vorschriften der StaatIichen Bauauficht der DDR /16/ und /24/.

Nachfolgend wird der wesentliche Inhalt der neuen Vorschrift dargestellt. An elnem Beispiel soll die Berechnung der inneren Stabilitat erlautert werden. 
Vorschrift "Bewehrte Erde - horizontal bewehrte Grindungspolster"

Die in der DDR geltenden Bestimmungen fordern den Nachweis der Tragfahigkeit des Baugrundes bei Belastung durch Flächenfundamente nach /18/, ab 1986 nach /23/. Dieser Standard sollte auch die Grundlage bilden fü den Nachweis der Tragfuhigkeit beim Binsatz tragfahigkeitserhöhender Konstruktionen. Die Vorschrift stutzt sich deshalb beim Tragfahigkeitsnachweis auf diesen Standard und erweitert seine Anwendbarkeit auf bewehrte Grindungspolster. Das gleiche gilt sinngema $B$ fur den Setzungsnachwe1s /17/. Die ersten drei Abschnitte in 131/, auf die hier nicht eingegangen wird, beinhalten den Geltungsbereich, Begriffe, Formelzeichen und Grundsätze. In Abschnitt 4., "Bauelemente", wird als Verfullmaterial kohäsionsloses Lockergestein mit Grenzwerten fur Kornungsanteile gefordert. Fur Baugrund und Bewehrung gilt $M_{0} / R_{1,0} \leqq 50$, um abzusichern, daß die Zerreißkraft der Bewehrung $\mathrm{R}_{1,0}$ fur $1 \mathrm{~m}$ breiten Streifen bei maximal $20 \%$ Bruchdehnurg durch entsprechend weichen Baugrund $\left(M_{0}=\right.$ Odometermodul) aktiviert werden kann. Als Reibung zwischen der Bewehrung und dem Verfullstoff darf $\mu=0,5$ fur Stahl und $\mu=\tan (0,8 \emptyset)$ fur geotextile Bewehrung ( $\emptyset=$ Winkel der inneren Reibung des Verfullmaterials) angenommen werden. Die Bewehrungsformen sind streifen- und stabförmig, flächenhaft geschlossen oder gitterartig. Bezüglich des Korrosionsschutzes wird auf bestehende Vorschriften, z. B. /16/, verwiesen.

Im Abschnitt 5., "Bauliche Durshbildung" (konstruktive Gestaltung), sind allgemeine Konstruktionsprinzipien genannt. Für streifen- oder stabförmige Bewehrungsformen wird deren Lage parallel zur schmalen Fundamentseite gefordert, wenn $\mathrm{B} / \mathrm{L} \leqq 0,2$ (Streifenfundament) ist. Eine kreuzweise oder fläshenhafte Bewehrung ist fur $\mathrm{B} / \mathrm{L}>0,2$ ( $\mathrm{B}=$ Breite, $\mathrm{L}=$ Länge des Fundamentes) erforderlich. Die Anzahl der Bewehrungslagen ist abhrngig von der vorhandenen Fundamentlast oder umgekehrt ist die Trägfahigkeit abhangig von der Anzahl der angenommenen Bewehrungslagen zu berechnen (stehe Belsplel). Bestimmung der Vertikalabstande $\Delta \mathrm{H}$ zwischen den Lagen:

$$
\mathrm{H}=\text { konst. }=0,15 \mathrm{~m} \text { bis } 0,40 \mathrm{~m} \leqq 0,5 \mathrm{~B}
$$


Ermittlung der Bewehrungslängen (Bild 54):

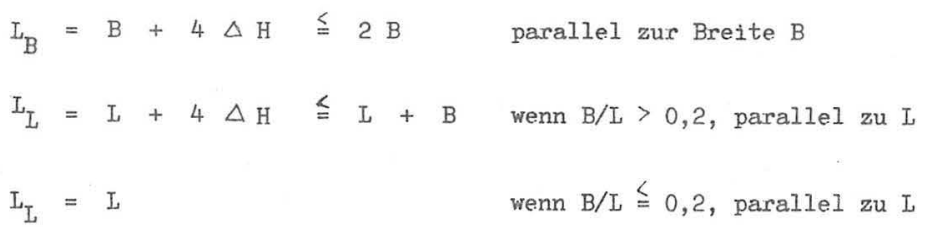

Die Abmessungen der Baugrube sind aus Bild 59 ersichtlich. Die Baugrubentiefe ist zu ermitteln nach:

$t_{p, \min }=3,5 \Delta H \leqq t_{p} \leqq t_{p, \max }=(B / 2) \tan \left(45^{\circ}+\varnothing / 2\right)$

$t_{p, \max }$ entspricht der Gleitkeiltiefe bei Vertikalbelastung, siehe Bild 60 .

Für das Polster wird eine lagenweise Verdichtung des Verfullstoffes bis $95 \%$ Standarddichte (Proctordichte) gefordert. Abschnitt 6. "Erdstatische Berechnungen" enthält Detailforderungen zu den Sicherheitsnachweisen. Fü die Nachweise der äußeren Stabilität werden als Grüdungssohle die Unterseite des Polsters und als Fundamentbreite und -länge die entsprechenden Bewehrungslängen angenommen. Diese Annahme gilt gleichzeitig fur die Berechnung der Tragfähigkeit, Geländebruch- und Gleitsicherheit nach den bestehenden Vorschriften und Standards.

Die Berechnung der Setzungen wirde nach dieser Verfahrensweise zu unreal hohen und nicht mit experimentellen Ergebnissen übereinstimmenden Werten fuhren. Deshalb besteht die Vorstellung, auf der Grundlage einer Arbeit von PIETSCH /32/ das Berechnungsverfahren /17/ zu modifizieren. Vergleichsrechnungen ergaben, daß damit plausible setzungswerte eshalten werden, die auch die Abhängigkeit der Setzungen von der Bewehrungsfestigkeit und der Bewehrungsdichte richtis widerspiegeln.

Der Nachweis der inneren Stabilität beginnt mit der Berechnung der erforderlichen Bewehrungskräfte $\sum z_{i}$, ausgehend von der vorhanderen, aufzunehmenden Last $N_{\text {vorh }}$ und der Tragfähigkeit $R_{f, p} / \eta_{s}$ des unbewehrten Polsters:

$1,8 \sum z_{i}=N_{\text {vorh }}-R_{f, p^{\prime} \eta s}$ 


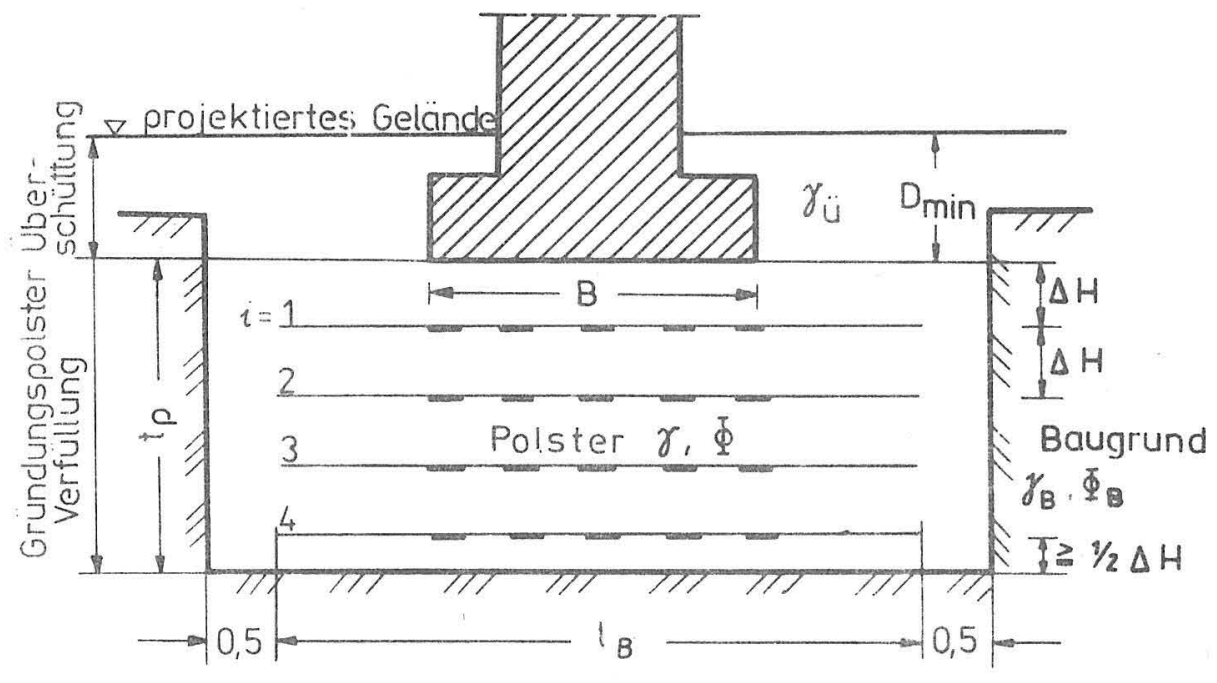

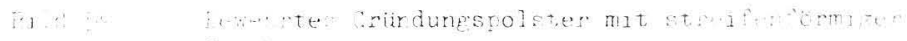
Fewet:म? है

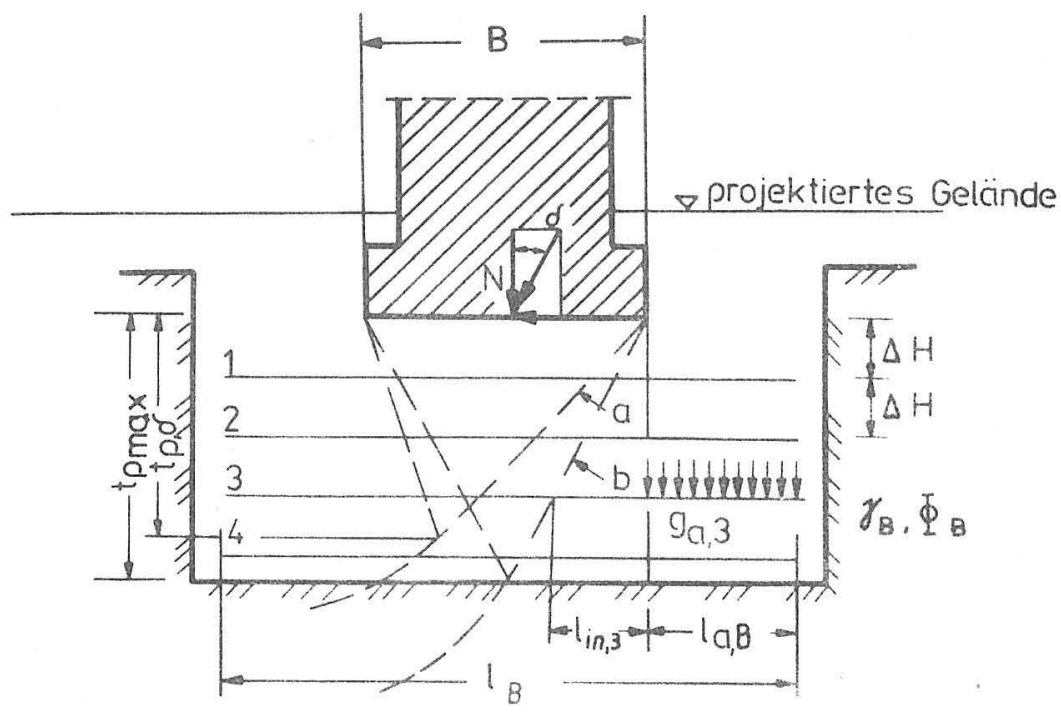


$\mathrm{R}_{\mathrm{f}, \mathrm{p}^{\prime}} \eta_{\mathrm{s}}$ wird nach /23/ fur den Baugrund ohne Polster berechnet, aber die tragfahigkeitserhöhende Wirkung des unbewehrten Polsters dursh Multiplikation der Tragfahigkeitsbeiwerte $\mathrm{N}_{b}, \mathrm{~N}_{\mathrm{g}}, \mathrm{N}_{\mathrm{c}}$ nach /23/ mit Korrekturfaktoren $\mathrm{k}_{\mathrm{B}}$, $\mathrm{k}_{\mathrm{g}}, \mathrm{k}_{\mathrm{c}}$ in Bild 61 berucksichtigt. Die Korrekturfaktoren gelten für

$t_{p, \mathcal{S}}=\bar{B} \frac{\tan \emptyset+\cos \phi_{1}}{1+\cot ^{2} \phi_{1}}, \phi_{1}$ nach /23/

Wird eine geringere Polsterdicke, $t_{p}<t_{p, \delta}$, gewählt, dann sind die Werte $\bar{k}$ statt $k$ zu verwenden. Dabei gilt:

$\bar{k}=1+(k-1) t_{p} / t_{p, \delta}$

Fur den Nachweis der inneren Stabilität wird die Berechnung der Sicherheit

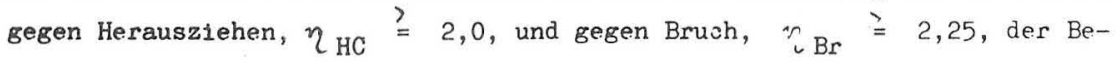
wehrung gefordert. Zum Beispiel gilt bei einer flachenhaften Bewehrung für die 1-te Bewehrungslage

$Z_{1, c r}=2\left(\frac{N_{\text {vorh }}}{\bar{B}} I_{\text {in, } i}+B_{a, i} I_{a, B^{L}}\right) \mu / \eta_{\text {HC }}$

$\bar{B}=$ wirksame Fundamentbreite nach /23/. Der Summand $g_{a}$, i $l_{a}, B$ kann im Regelfall vernachlässigt werden

$1_{1 n, 1}=\frac{i \Delta H}{\tan \left(45^{\circ} \quad \varnothing / 2\right)}$

$1_{a, B}=\left(1_{B}-B\right) / 2$

$g_{a, 1}=1 \Delta H \gamma+\gamma_{u} D_{\min }$ 


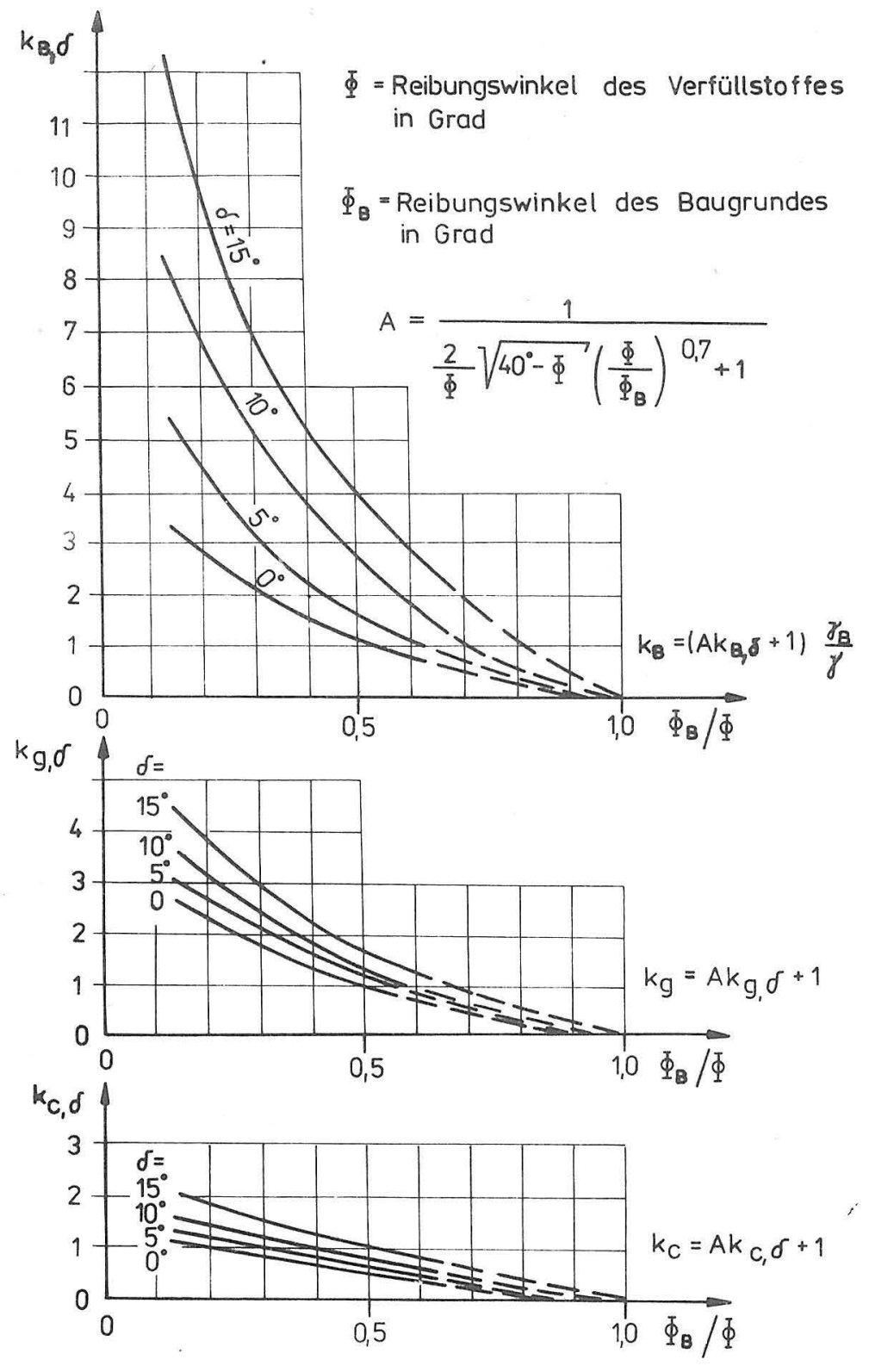

bortektorfahtrrer 
Mit $i=1,2, \ldots \leqq n$ ist schrittweise zu prufen:

$\frac{5 z_{i}}{n+1-i}=z_{i}, \approx r$

womit die Sicherheit gegen Herausziehen erfullt ist. Für die Anzahl der Bewhrungslagen, die gegen Herausziehen sicher sind, ist ferner die Sicherheit gegen Bruch zu berechnen.

$\frac{\Sigma Z_{i}}{n+1-i}=\frac{z_{B r}}{\eta_{B r}}$

$\mathrm{Z}_{\mathrm{Br}}=\mathrm{R}_{1,0} \mathrm{~L}$

\subsection{Berechnungsbeispiel}

3.2.1. Nachweis der inneren Stabilitat

Baugrund:

Aueton, weich, $I_{c}=0,7 ; \quad \emptyset_{B}=20^{\circ} ; \quad s=10 \mathrm{kN} / \mathrm{m}^{2} ;$

$\gamma_{\mathrm{B}}=17 \mathrm{kN} / \mathrm{m}^{3} ; \gamma_{\mathrm{B}}{ }^{\prime}=8,0 \mathrm{kN} / \mathrm{m}^{3}$ (Auftrieb), $\mu_{0}=2000 \mathrm{kN} / \mathrm{m}^{2}$

Tragfahigkeits- und Lastneigungsbeiwerte nach / $3 /$ :

$\mathrm{N}_{B}=2,0 ; \mathrm{N}_{\mathrm{C}}=6,4 ; \mathrm{N}_{\mathrm{s}}=14,8 ; \mathrm{i}_{\mathrm{B}}=0,43 ; i_{\sigma}=0,67 ; i_{\mathrm{C}}=0,64$

Last:

$N_{n}=200 \mathrm{kN}$, Exzentrizitat $e_{\mathrm{B}}=0,2 \mathrm{~m}, \quad[-10)^{2}, \quad ?_{\mathrm{s}}=2,0$ 
Fundament:

$B=1,0 \mathrm{~m} ; \quad D_{\min }=1,0 \mathrm{~m} ;$ Streifenfundament

Bewehrung :

Geotextil, $R_{1,0}=80 \mathrm{kN} / \mathrm{m}$, flächenhaft verlegt

$\Delta H=0,18 \mathrm{~m}, \quad 5$ Lagen, $\mu=\tan 0,8 \cdot 36^{\circ}=0,59$

Polster:

$t_{p}=5,5 \Delta H=0,99 \mathrm{~m}$, Sand, pitteldicht, $I_{D}=0,5, \quad \varnothing=38^{\circ}$

$\gamma=19,5 \mathrm{kN} / \mathrm{m}^{3}$

Bereshnungen:

$t_{p, \max }=\frac{1,0}{2} \tan \left(45^{\circ}+38^{\circ} / 2\right)=1,03 \mathrm{~m}>0,99 \mathrm{~m}=\mathrm{t}_{\mathrm{p}}$

$M_{0} / R_{1,0}=2000 / 80=25<50, \quad I_{B}=B+4 \Delta H=1,72 \mathrm{~m}$

$\bar{B}=B-2 e_{B}=0,6 \mathrm{~m}$,

Bruahkeiltiefe: $t_{p, \delta}=0,6 \frac{\tan 38^{\circ}+\cos 74,4^{\circ}}{1+\cos ^{2} 74,4^{\circ}}=0,58 \mathrm{~m}$

$t_{p, \delta} / \triangle H=3,2, d . h . n=3$ Bewehrungslagen werden vom Bruchkeil gesshnitten. 
Korrekturfaktoren nach Bild 61:

$$
\begin{aligned}
A=1 /\left(\frac{2}{38} \sqrt{40-38}\left(\frac{38}{20}\right)^{0,7}+1\right)=0,895 \\
k_{B}=(2,5 \cdot 0,895+1) \frac{8,0}{19,5}=1,3 ; \quad k_{g}=1,25 \cdot 0,895+1=2,1 ; \\
k_{0}=0,8 \cdot 0,895+1=1,7
\end{aligned}
$$

Grenztragfahigkeit nash /23/ und Bild 61 :

$$
\begin{aligned}
& \mathrm{R}_{\hat{1}^{\prime}, p, B}=\bar{B} \bar{B} \gamma_{B}{ }^{\prime} N_{B} k_{B} i_{B} \cdot 1 \mathrm{~m}=0,6 \cdot 0,6 \cdot 8,0 \cdot 2,0 \cdot 1,3 \cdot 0 \cdot 48= \\
& 3.6 \mathrm{kN} \\
& \mathrm{F}_{\mathrm{f},},=\overline{\mathrm{B}} \mathrm{D}_{\min } \gamma_{\mathrm{B}} \mathrm{N}_{\mathrm{g}} \mathrm{k}_{\mathrm{g}}{ }_{\mathrm{g}} \cdot 1 \mathrm{~m}=0,6 \cdot 17,0 \cdot 6,4 \cdot 2,1 \cdot 0,67= \\
& 91,8 \mathrm{kN} \\
& 96,6 \mathrm{kN}
\end{aligned}
$$

Prufung der Sicherheit gegen Herausziehen:

$$
\begin{aligned}
& \sum z_{i}=\frac{1}{1,8}\left(200-\frac{192,0}{2,0}\right)=57,8 \mathrm{kN} \\
& 1_{\text {in, } 1}=1.0,18 / \tan \left(45^{\circ}+38^{\circ} / 2\right)=0,1 \mathrm{~m} ; \\
& 1_{\text {in, } 2}=0,20 \mathrm{~m} \\
& 1_{\text {in, } 3}=0,30 \mathrm{~m}
\end{aligned}
$$




$$
z_{1, \text { er }}=2 \cdot \frac{200}{0,6} \cdot 0,10 \cdot \frac{0,59}{2,0}=19,7 \mathrm{kN}
$$

$\frac{\sum z_{i}}{n-i+1}=\frac{57,8}{3-1+1}=19,3 \mathrm{kN}<19,7 \mathrm{kN}=z_{1}$, ar , d. h. die 1. Lage Bewehrung ist herausziehsicher und damit auch die beiden folgenden Lagen.

Sollte dieses Kriterium nicht erfült sein, muß die gleiche Prüung für die 2. Lage durchgeführt werden. Diese Rechnung soll hier, obwohl für das angenommene Beispiel nisht notwendig, durshgeführt werden:

$z_{2, \text { or }}=2 \cdot \frac{200}{0,6} \cdot 0,20 \cdot \frac{0,59}{2,0}=39,3 \mathrm{kN}$ $\frac{\Sigma Z_{i}}{n-i+1}=\frac{57,8}{3-2+1}=28,9 \mathrm{kN}<39,3 \mathrm{kN}=z_{2, \text { ar }}$, d. h. aush die 2. und die 3. Lage sind herausziehsiaher.

Prüfung der Sicherheit gegen Bewehrungsbrush:

$\frac{\sum z_{i}}{n-1+1}=\frac{57,8}{3-1+1}=19,3 \mathrm{kN}<\frac{R_{1,0}}{\eta_{B r}}=\frac{80}{2,25}=35,6 \mathrm{kN}$, d. $\mathrm{h}$. die Bruchsicherheit ist vorhanden. Damit ist die Tragfähigkeit des bewehrten Polsters insgesamt gewährleistet.

Vergleish:

Tragfahigkeit des Polsters ohne Bewehrung

$R_{f, p} / \eta_{s}=192,0 / 2,0=96,0 \mathrm{kN}<\mathrm{N}_{\mathrm{n}}=200 \mathrm{kN}$

ist nisht ausreishend! 
Tragfähigkeit des Baugrundes ohne Polster:

$R_{f}=3,6 / 1,3+91,8 / 2,1+96,6 / 1,7=103,3 \mathrm{kN}$

$\mathrm{R}_{\mathrm{f}} / \eta_{\mathrm{S}}=103,3 / 2,0=51,7 \mathrm{kN}<96,0 \mathrm{kN}<200 \mathrm{kN}$

ist nicht ausreichend!

3.2.2. Bewehrtes Gründungspolster unter einem Brizkenwiderlager (Fortsetzung des Beispiels in Absohnitt 2.1.1.3.)

Berechnung nach /31/, Bild 15

Verfullmaterial: Sand; $\gamma=18,5 \mathrm{kN} / \mathrm{m}^{3} ; \quad \phi=36^{\circ} ; \quad 0=\cdot 0$

Bewehrung: flächenhaft aus Geotextil

$$
\begin{aligned}
& \mathrm{R}_{1,0}=80 \mathrm{kN} / \mathrm{m} ; \mu=\tan \left(0,8 \cdot 36^{\circ}\right)=0,55 \\
& \frac{\mathrm{M}_{0}}{\mathrm{R}_{1,0}}=\frac{2000}{80}=25<50
\end{aligned}
$$

Gewählt $\Delta H=0,25 \mathrm{~m}$

$$
n=4
$$$$
t_{p}=(n+0,5) \Delta n=4,0,25=1,12 \mathrm{~m}
$$$$
t_{p, \min }=3,5 \Delta H=3,5 \cdot 0,25=0,88 \mathrm{~m}<1,12 \mathrm{~m}=t_{\mathrm{p}}
$$$$
t_{p, \max }=\frac{B}{2} \tan \left(45^{\circ}+\phi / 2\right)=\frac{5,0}{2} \tan 63^{\circ}=4,91 \mathrm{~m}>1,12 \mathrm{~m}=t_{\mathrm{p}}
$$ 
$I_{B}=B+4 \angle H=5,00+4 \cdot 0,25=6,00 m<10,0 m=2 B$

$I_{L}=\mathrm{L}+4 \Delta \mathrm{H}=6,23+4 \cdot 0,25=7,23 \mathrm{~m}<11,26 \mathrm{~m}=\mathrm{L}+\mathrm{B}$

Nachweis der inneren Stabilitat des Polsters

nach /31/, Abschnitt 6.3.

Obwohl $\mathrm{B} / \mathrm{I}>0,2$, wird der Nachweis hier nur in Richtung parallel zur Fundamentbreite geführt. Parallel zur Fundamentlänge sind keine Horizontalkrufte wirksam, so daß eine wesentlich ginstigere Lastkombination entsteht. Wegen des geneigten Lastangriffs ist zunäihst nach /23/ zu rechnen:

$$
\begin{aligned}
\cot \phi_{L} & =\sqrt{\left(1+\tan ^{2} 36^{\circ}\right) \frac{\tan 36^{\circ}-\tan 8,12^{\circ}}{\tan 36^{\circ}+\tan 8,12^{\circ}}}-\tan 36^{\circ} \\
& =0,2865 ; \phi_{L}=74,01^{\circ}
\end{aligned}
$$

und nash /31/:

$t_{p, \delta}=4,32 \frac{\tan 36^{\circ}+\cos 74,01^{\circ}}{1+\cot 74,01^{\circ}}=3,36 \mathrm{~m}$

Für $\frac{\phi_{B}}{\phi}=\frac{20^{\circ}}{36^{\circ}}=0,56$ und $c^{\dagger}=\xi_{B}=8,12^{\circ}$ folgt aus Bild 56 :

$$
K_{B, \S}=1,90 ; \quad K_{a, \delta}=0,65
$$

$A=\frac{1}{\frac{\because}{36^{\circ}} \sqrt[7]{40^{\circ}-36^{\circ}}\left(\frac{36^{\circ}}{20^{\circ}}\right)^{0,7}+1}$ 


$$
\begin{aligned}
& \mathrm{K}_{\mathrm{B}}=(0,86 \cdot 1,90+1) \frac{17,0}{18,5}=2,42 \\
& \mathrm{~K}_{\mathrm{c}}=0,86 \cdot 0,65+1=1,56 \\
& / 31 /, \\
& \overline{\mathrm{K}_{\mathrm{B}}}=1+(2,42-1) \frac{1,12}{3,36}=1,47 \\
& \overline{\mathrm{K}_{\mathrm{C}}}=1+(1,56-1) \frac{1,12}{3,36}=1,19
\end{aligned}
$$

Daher:

$$
\begin{aligned}
\mathrm{R}_{\bar{B}, p} & =1840,42 \cdot 1,47=2705,42 \mathrm{kN} \\
\mathrm{R}_{\imath, p} & =3382,45 \cdot 1,19=4025,12 \mathrm{kN} \\
\mathrm{R}_{\mathrm{f}, \mathrm{p}} & =2705,42+4025,12=6730,54 \mathrm{kN} \\
\mathrm{R}_{\mathrm{p}} & =\frac{6730,54}{2}=3365,27 \mathrm{kN}<4054,58 \mathrm{kN}=\mathrm{N}_{\mathrm{vorh}} . \\
\mathrm{Z}_{i} & =\Gamma_{4054,58}-\frac{6730,54}{2,0}-\frac{1}{1,8}=382,95 \mathrm{kN}
\end{aligned}
$$

Nachweis piegen Herausziehen der Bewehrung

$$
\begin{aligned}
& 1_{\text {in, } 1}=\frac{1 \cdot 0,25}{\tan \left(45^{\circ}-35^{0} / 2\right)}=0,13 \mathrm{~m} ; \quad 1_{1 \mathrm{n}, 2}=0,25 \mathrm{~m} \\
& 1_{1 \mathrm{n}, 3}=0,38 \mathrm{~m} ; \quad 1_{1 \mathrm{n}, 4_{4}}=0,51 \mathrm{~m}
\end{aligned}
$$


$I_{a, B}=\frac{1}{2}(6,00-4,32)=0,84 \mathrm{~m}$

$9_{a, 1}=1 \cdot 0,25 \cdot 18,5=4,62 \mathrm{kN} / \mathrm{m}^{2} ; \quad 9_{\mathrm{a}, 2}=9,25 \mathrm{kN} / \mathrm{m}^{2}$

$\vartheta_{\mathrm{a}, 3}=13,88 \mathrm{kN} / \mathrm{m}^{2} ; \quad g_{\mathrm{a}, 4}=18,5 \mathrm{kN} / \mathrm{m}^{2}$

$z_{1, c r}=2\left(\frac{4054,58}{4,32} \quad 0,13+4,62 \cdot 0,84 \cdot 6,23\right) \quad 0,55 \frac{1}{2,0}=$ $80,41 \mathrm{kN}$

$\mathrm{z}_{2, \text { cr }}=155,68 \mathrm{kN}$

$\frac{\mathrm{Z}_{1}}{4-1+1}=\frac{382,95}{4}=95,74 \mathrm{kN}>80,41 \mathrm{kN}=\mathrm{Z}_{1, \text { ar }}$

$\frac{\mathrm{z}_{1}}{4-2+1}=\frac{382,95}{3}=127,65 \mathrm{kN}<155,68 \mathrm{kN}=\mathrm{Z}_{2, \text {, }}$

Der Nachweis ist für $i=2<4=n$ erfullt.

Nachweis gegen Bruch der Bewehrung

$\frac{Z_{i}}{4-2+1}=\frac{382,95}{3}=127,65 \mathrm{kN}<\frac{806,23}{2,25}=221,51 \mathrm{kN}$

Der Nachweis ist erfullt. 
Nachweis der außeren Stabilitat des Polsters

(nach /31/ in Verbindung mit /23/)

$$
\text { Rechteckfundament } \quad \begin{aligned}
B & =I_{B}=6,00 \mathrm{~m} \\
L & =I_{L}=7,23 \mathrm{~m} \\
& =0 ; D_{\min }=t p=1,12 \mathrm{~m}
\end{aligned}
$$

Lasten dem aus Bauwerk (bei voller Verkehrsbelastung einschlieBlich Bremsvorgängen)

$$
\begin{aligned}
& \mathrm{N}_{\text {vorh }}=4054,58+6,00 \cdot 7,23 \cdot 1,12 \cdot 18,5=4953,41 \mathrm{kN} \\
& \mathrm{T}_{\text {vorh }}=578,62 \mathrm{kN}
\end{aligned}
$$$$
\tan \delta=\frac{578,62}{4953,41}=0,1168 ; \quad \delta=6,66^{\circ} ; \quad g=1,12 \cdot 17,0=
$$$$
19,04 \mathrm{kN} / \mathrm{m}^{2}
$$$$
\text { e }=0,41 \mathrm{~m}
$$$$
\bar{B}=B-2 e=6,00-2 \cdot 0,41=5,18 \mathrm{~m}
$$$$
\overline{\mathrm{L}}=\mathrm{L}=7,23 \mathrm{~m}
$$

$$
\begin{aligned}
& \bar{A}=5,18 \cdot 7,23=37,45 \mathrm{~m}^{2} \\
& \frac{\bar{B}}{\bar{L}}=\frac{5,18}{7,23}=0,72
\end{aligned}
$$$$
N_{B}=2,04
$$$$
N_{B}=6,4 \quad \text { siehe Abschnitt 2.1.1.3. }
$$$$
N_{c}=14,48
$$ 


$$
\begin{aligned}
& i_{\bar{B}}=\left(1-0,57 \tan 6,66^{\circ}\right)^{7}=0,62 \\
& i_{g}=\left(1-0,70 \tan 6,66^{\circ}\right)^{3}=0,77 \\
& i_{c}=\left(1-0,59 \tan 6,66^{\circ}\right)^{4}=0,75 \\
& s_{\bar{B}}=1-0,25 \cdot 0,72=0,82 \\
& \mathrm{~s}_{\mathrm{g}}=1+1,8 \cdot 0,72 \sin 20^{\circ}=1,44 \\
& \mathbf{s}_{2}=1+0,3 \cdot 0,72=1,22 \\
& \mathrm{R}_{\overline{\mathrm{B}}}=37,45 \cdot 5,18 \cdot 17,0 \cdot 2,04-0,62 \cdot 0,82=3420,32 \mathrm{kN} \\
& R_{g}=37,45 \cdot 19,04 \cdot 6,4 \cdot 0,77 \cdot 1,44=5060,02 \mathrm{kN} \\
& R_{2}=37,45 \cdot 10,0 \cdot 14,84 \cdot 0,75 \cdot 1,22=5085,19 \mathrm{kN} \\
& R_{f}=3420,32+5060,02+5085,19=13565,53 \mathrm{kN} \\
& \mathrm{R}=\frac{13565,32}{2,0}=6782,76 \mathrm{kN}>4953,41 \mathrm{kN}=\mathrm{N}_{\text {vorh }}
\end{aligned}
$$

Gleitsicherheitsnachweis nach /33/

Der Nashweis wird für die Fuge zwisshen dem Stutzbauwerk und dem Grundungspolster gefürt.

$\tan 0=\tan \hat{i}_{B}=0,1427<0,40$ 


\section{Geländebruchsicherheit}

Der Nachweis ist hier nicht erforderlich.

Formänderungsnashweis nach /17/

Es sind die Setzungen im Untergrund nachzuweisen. Im verdichteten Grundungspolster wird vorausgesetzt, daß nur vernachlässigbar kleine Setzungen entstehen, ebenso werden die Eigensetzungen im Stützbauwerk als vernachlässigbar klein angenommen. Der Nachweis erfolgt unter der Annahme eines Zweischichtensystems (unzusammendrückbare Schicht des Polsters über dem zusammendrückbaren Untergrund). Um relative Durchbiegungen angeben zu können, werden Setzungsnachweis für zwei Punkte, unterhalb der Außenhaut des Stützbauwerkes liegend, gefuhrt. Ohne Berlucksichtigung der Verkehrslast ist in der Gründungssohle des Stützbauwerks

$\mathrm{N}=3518,17 \mathrm{kN} ; \quad$ e $=0,03 \mathrm{~m}$.

Hieraus ergibt sich eine trapezförmige Spannungsverteilung mit

$$
\begin{aligned}
& \sigma_{\mathrm{N} 1}=117,34 \mathrm{kN} / \mathrm{m}^{2} \text { (unter Außenhaut) und } \\
& \sigma_{\mathrm{N} 2}=108,54 \mathrm{kN} / \mathrm{m}^{2} \text { (unter den Bewehrungsbandenden). }
\end{aligned}
$$

Fur den Eckpunkt des Bauwerks (Außenhautende) ergibt sich

$$
s_{1}=0,076 \mathrm{~m}
$$

und unter dem Mittelpunkt der Außenhaut

$$
\begin{aligned}
& s_{2}=0,102 \mathrm{~m} \\
& \mathrm{f} / 1=\frac{0,102-0,076}{6,23}=0,004<\underline{0,005}
\end{aligned}
$$

Im Abschnitt 2.1.1.3. konnte nur eine zu geringe Tragfahigkeit des Untergrundęs nachgewiesen werden. Vorstehende Berechnungen weisen nach, daß die Tragfahigkeit bei Anordnung eines Grindungspolsters ausreichend ist. 